\title{
miR-139 regulates the proliferation and invasion of hepatocellular carcinoma through the WNT/TCF-4 pathway
}

\author{
WEI GU ${ }^{1,2}$ XIAOMEI $\mathrm{LI}^{2}$ and JUE WANG ${ }^{1}$ \\ ${ }^{1}$ Institute of Biomedical Engineering, School of Life Science and Technology, and ${ }^{2}$ College of Medicine, \\ Xi'an Jiaotong University, Xi'an, Shaanxi 710049, P.R. China
}

Received July 18, 2013; Accepted September 5, 2013

DOI: $10.3892 / o r .2013 .2831$

\begin{abstract}
RNAs (miRNAs) are key post-transcriptional regulators of gene expression in hepatocellular carcinoma (HCC), but their specific roles and functions have yet to be fully elucidated. In the present study, a significant downregulation of miR-139 expression was demonstrated in HCC samples and HCC cells using quantitative PCR (qPCR). Upregulation of miR-139 in vitro, attenuated HCC cell growth, migration/invasion and induced apoptosis. Based on computational and expression analysis, we noted that miR-139 can control the expression of T-cell factor-4 (TCF-4) as a target gene. A reporter assay with the 3'UTR of TCF-4 cloned downstream of a luciferase gene showed decreased luciferase activity in the presence of miR-139, providing strong evidence that miR-139 is a direct regulator of TCF-4. Furthermore, we observed that restoration of $\mathrm{TCF}-4$ activity resulted in effects that were similar to those following transfection of the miR-139 inhibitor into HCC cells. Finally, mechanistic investigation revealed that the overexpression of miR-139 suppressed the $\beta$-catenin/ TCF-4 transcriptional activity by targeting TCF-4. In conclusion, our study demonstrates that miR-139 downregulation is common in HCC and that overexpression of miR-139 expression inhibits cell proliferation and invasion, suggesting that miR-139 may provide a therapeutic strategy for the treatment of HCC patients.
\end{abstract}

\section{Introduction}

Hepatocellular carcinoma (HCC) is one of the most common types of primary cancers in the world (1), ranking as the sixth most prevalent cancer and the third most frequent cause of cancer-related mortality (2). HCC has a poor 5-year survival

Correspondence to: Professor Jue Wang, Institute of Biomedical Engineering, School of Life Science and Technology, Xi'an Jiaotong University, No. 28 Xianning West Road, Xi'an, Shaanxi 710049, P.R. China

E-mail: juewang818@126.com

Key words: miR-139, hepatocellular carcinoma, T-cell factor-4, $\beta$-catenin rate of $\sim 7 \%$ despite treatment (3). Potentially curative therapies, including liver transplantation and surgical resection, are only applied to a minority of patients due to a high rate of advanced-stage disease at the time of diagnosis and the lack of suitable organ donors. Other regional treatments may be beneficial for unresectable HCC, but local failure or recurrences are frequent, and the long term survival rate remains poor. The development of innovative, targeted therapies may represent an alternative for the treatment of these cancers.

microRNAs (miRNAs) are an evolutionarily conserved novel class of endogenous small non-coding RNAs present extensively in eukaryotes, that were first discovered in a 1994 investigation into eelworms $(4,5)$. Most of the miRNAs are transcribed by RNA polymerase II as primary miRNAs and are processed by the RNase III enzymes Drosha and Dicer to produce 21- to 23-nucleotide double-stranded RNA duplexes $(6,7)$. These smaller RNAs are then exported to the cytoplasm by Exportin-5, where they are subsequently processed into mature miRNAs by Dicer (8). The mature miRNAs are loaded into the miRNA-induced silencing complex, where they recognize their target protein-coding mRNAs to inhibit mostly mRNA translation or degradation by base pairing to complementary sequences within the $3^{\prime}$ untranslated region (3'UTR) (9). With respect to miRNA functions, they play pivotal roles in pathophysiological processes such as apoptosis, cell differentiation, cell proliferation and organ development (10). Functionally speaking, several human diseases have now been associated with dysregulated miRNA expression.

The oncogenic activity associated with Wnt signaling was first recognized by the ability of Wnt-1 to promote tumor formation when ectopically expressed in mouse mammary tissue $(11,12)$. Paradoxically, Wnt ligands themselves have never been formally implicated in contributing to human cancers. However, 2 tumor suppressors in the Wnt pathway, Axin and APC, and the proto-oncogene $\beta$-catenin, are commonly mutated in a variety of human neoplasms $(12,13)$. Mutations in these genes deregulate the Wnt pathway by stabilizing $\beta$-catenin. Normally associated with cadherins at the plasma membrane, the uncomplexed form of $\beta$-catenin is stabilized in response to Wnt signaling and forms complexes with transcription factors of the TCF/LEF family (14). These HMG domain-containing transcription factors contain high affinity binding sites for $\beta$-catenin in their extreme $\mathrm{N}$-terminal 
regions (15), and the bound $\beta$-catenin recruits various accessories essential for gene activation (16-18). A hallmark of Wnt signaling in cancer is the stabilization and/or mislocalization of $\beta$-catenin, which presumably exerts its oncogenic effect by exacerbating the transcription of growth controlling genes.

TCF-4 promotes initiation and progression of many types of cancers. TCF- 4 binds to $\beta$-catenin to transactivate Wnt target genes (19). However, in the absence of $\beta$-catenin, TCF-4 becomes a repressor of transcription of those target genes via binding to corepressors (20). This characteristic may explain the apparently inconsistent findings that TCF-4 can either promote or repress breast cancer progression in different cellular contexts. We previously showed that TCF-4 binds to a metastasis-inducing DNA sequence (21). This results in a reduction in the amount of TCF-4 available to regulate the expression of endogenous genes, leading to a decrease in metastatic development (22).

The present study aimed to investigate whether miRNAs are involved in the regulation of HCC apoptosis. In this study, we focused on miR-139. Using qRT-PCR techniques, both our HCC patient tissue samples and cancer cell lines had lower miR-139 expression levels, as predicted. Furthermore, overexpression of miR-139 was involved in decreased HCC proliferation and invasion and induction of apoptosis. TCF-4 was found to have binding sites for miR-139 in the 3'UTR region. We demonstrated that TCF-4 is a direct target of miR-139. These findings suggest that miR-139 may act as a biomarker in HCC and that upregulation of miR-139 is a possible therapeutic approach for HCC that merits further evaluation.

\section{Materials and methods}

Tissue samples and clinical data. Informed consent was obtained for the use of 50 human HCC tissue samples from adult patients diagnosed with $\mathrm{HCC}$ at the First Affiliated Hospital of Medical College of Xi'an Jiaotong University, Xi'an, China. This study was approved by the hospital institutional review board, and written informed consent was obtained from all patients.

Cell culture and transfection. The human embryonic kidney cell line (HEK293), the human HCC cell lines, HepG2, Hep3B, SMMC7721, and the normal liver cell line HL7702, were obtained from the American Type Culture Collection (Rockville, MD, USA), and cultured in Dulbecco's modified Eagle's medium (DMEM) supplemented with $10 \%$ fetal bovine serum (FBS) and $100 \mathrm{IU} / \mathrm{ml}$ penicillin/streptomycin in a $37^{\circ} \mathrm{C}$ humidified incubator with $5 \% \mathrm{CO}_{2}$.

Plasmids and oligonucleotides. The hsa-miR-139 inhibitor and mimics were chemically synthesized and purified by highperformance liquid chromatography (GenePharma, Shanghai, China). The hsa-miR-139 mimics included the following: sense, 5'-GGA GAC GCG GCC CUG UUG GAG U-3' and antisense, 5'-UCC AAC AGG GCC GCG UCU CCU C-3'. The hsa-miR-139 inhibitor was 5'-CUU CCA ACA GGG CCG CGU CUC C-3'. The microRNA mimic controls were 5'-GGA GAC GCG GCC CUG UUC GAG U-3' and 5'-TCG AAC AGG GCC GCG UCU CCU C-3'. The microRNA inhibitor control was 5'-UUG UCA CAA ACU AAA GUA CUG-3'. The miR-139 mimic, inhibitor or their respective control $(40 \mathrm{nM})$ was transfected into cells using Lipofectamine ${ }^{\mathrm{TM}} 2000$ reagent (Invitrogen Life Technologies, Carlsbad, CA, USA) according to the manufacturer's instructions in serum-free conditions for $6 \mathrm{~h}$ before replacing with complete medium. The efficiency of transfection was determined using a Leica DMIRE2 microscope system (Leica Microsystems, Montreal, QC, Canada). RNA and protein were collected 48 and $72 \mathrm{~h}$ after transfection, respectively.

$q R T-P C R$. To assess the expression of miRNA-139 in HCC tissues and cell line, qRT-PCR was carried out using the ABI 7500 Real-Time PCR system (Applied Biosystems, Foster City, CA, USA). For detecting mature miR-139, reverse transcription and quantitative PCR were performed using the Hairpin-it $^{\mathrm{TM}}$ miRNAs qPCR Quantitation kit (GenePharma). U6 small nuclear RNA (snRNA) expression was assayed for normalization. The thermal cycling conditions for miR-139 and U6 snRNA consisted of $95^{\circ} \mathrm{C}$ for $3 \mathrm{~min}, 40$ cycles of $95^{\circ} \mathrm{C}$ for $12 \mathrm{sec}$ and $62^{\circ} \mathrm{C}$ for $1 \mathrm{~min}$. To detect TCF- 4 mRNA expression, the RevertAid ${ }^{\mathrm{TM}}$ First Strand cDNA Synthesis kit (MBI Fermentas, Burlington, ON, Canada) and SYBR ${ }^{\circledR}$ Premix ExTaq ${ }^{\mathrm{TM}}$ II (Takara Bio, Inc., Dalian, China) were used. TCF-4 (forward primer, 5'-GGC TAT GCA GGA ATG TTG GG-3' and reverse primer, 5'-GTT CAT GTG GAT GCA GGC TAC-3'), and reference gene GAPDH (forward primer, 5'-CTG GGC TAC ACT GAG CAC C-3' and reverse primer, 5'-AAG TGG TCG TTG AGG GCA ATG-3') were generated and optimized to an equal annealing temperature of $60^{\circ} \mathrm{C}$. The conditions for the TCF-4 and GAPDH reaction mixtures were $95^{\circ} \mathrm{C}$ for $2 \mathrm{~min}, 40$ cycles of $95^{\circ} \mathrm{C}$ for $15 \mathrm{sec}$ and $60^{\circ} \mathrm{C}$ for $1 \mathrm{~min}$ and finally $95^{\circ} \mathrm{C}$ for $15 \mathrm{sec}, 60^{\circ} \mathrm{C}$ for $1 \mathrm{~min}, 95^{\circ} \mathrm{C}$ for $15 \mathrm{sec}$ and $60^{\circ} \mathrm{C}$ for $15 \mathrm{sec}$. All experiments were performed in triplicate. Analysis of relative miR-139 or TCF-4 mRNA expression was performed using the comparative $\mathrm{C}_{t}$ method with U6 and GAPDH snRNA as endogenous controls, respectively.

Proliferation assay. Following transfection with miR-139, an MTT assay was used to quantitate the cell viability of human HCC cells. Each experiment was performed in triplicate. The absorbance values of each well were measured with a microplate spectrophotometer (Molecular Devices, Sunnyvale, CA, USA) at $450 \mathrm{~nm}$. The proliferation analysis of each group in each individual experiment and the whole experiment process were performed in triplicate.

Cell apoptosis analysis. For cell cycle analysis by flow cytometry (FCM), transfected and control cells in the log phase of growth were harvested by trypsinization, and washed with PBS. Nuclei of cells were then stained with Annexin V and PI for $30 \mathrm{~min}$. A total of $10^{4}$ nuclei was examined in a FACSCalibur flow cytometer (Becton-Dickinson, Franklin Lakes, NJ, USA). Experiments were performed in triplicate.

In vitro invasion assays. Transwell membranes coated with Matrigel (BD Biosciences, San Jose, CA, USA) were used to quantify in vitro HCC cell invasion. Transfected cells were plated at $5 \times 10^{4} /$ well in the upper chamber in serum-free medium. Medium containing 20\% FBS was added to the lower 

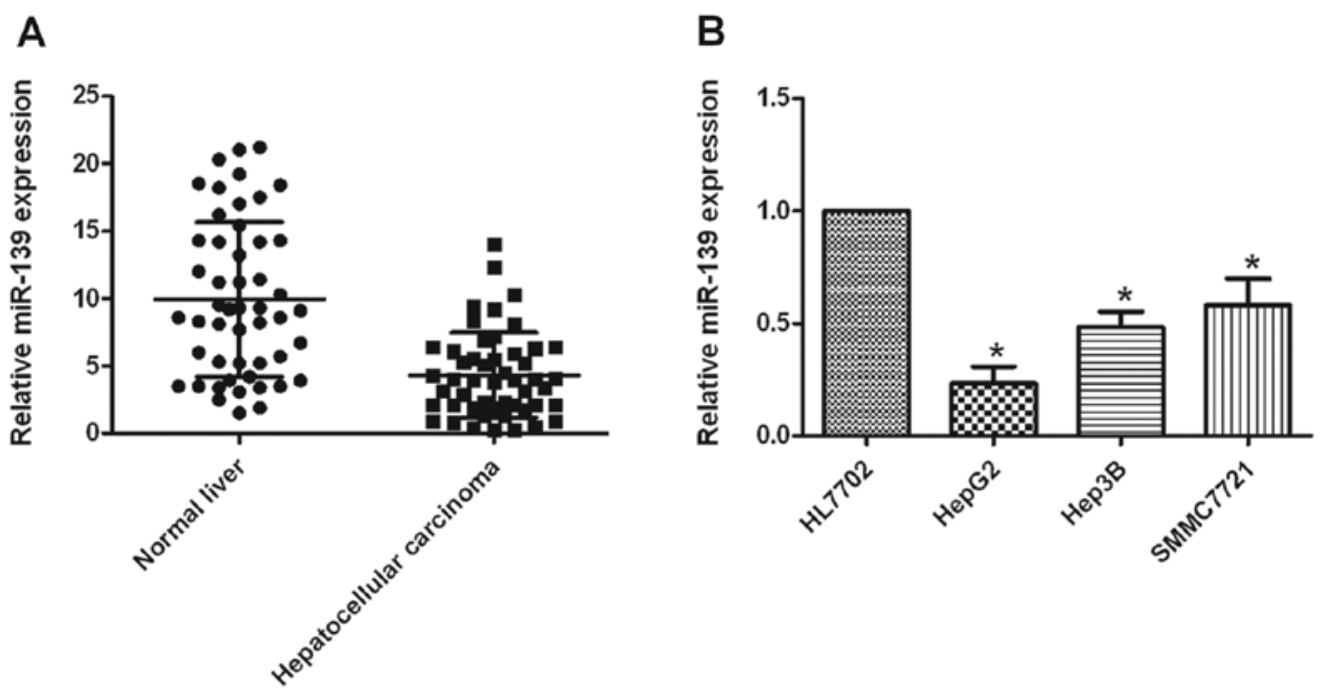

Figure 1. miR-139 exhibits high expression in the normal liver samples. (A) miR-139 expression in normal liver and HCC tissue samples as detected by qRTPCR.(B) qRT-PCR analysis showed that HepG2, Hep3B and SMMC7721 cell lines expressed lower levels of miR-139 when compared with the normal liver HL7702 cells. Data are expressed as the means \pm SD of 3 replicate. ${ }^{*} \mathrm{P}<0.05$.

chamber. Following a 24-h incubation, non-invading cells were removed from the top well with a cotton swab while the bottom cells were fixed with $3 \%$ paraformaldehyde, stained with $0.1 \%$ crystal violet, and photographed in 3 independent $\mathrm{x} 100$ fields for each well. The fold-change in migration was calculated relative to the blank control. The data are represented as the means \pm SD for 3 independent experiments.

Luciferase reporter assay. The 3'UTR of the human TCF-4 gene was PCR amplified using the following primers: TCF-4 forward, 5'-TCT AGA GGA TTA ACT AAT TGA AGG-3' and reverse, 5'-TCT AGA CAG TAA GAA CAT AAG AAC ATC T-3'. They were then cloned downstream of the Renilla luciferase stop codon in the pGL3 control vector (Promega Corporation, Madison, WI, USA), giving rise to the p'UTR-TCF-4 plasmids. These constructs were used to generate, by inverse PCR, the p3'UTRmut-TCF-4 plasmid (primers: forward, 5'-ATT TGG AGG CTA TTT CAA GTT GAA AAA AGG AAA TGT AAA GTA A-3' and reverse, 5'-TTA CTT TAC ATT TCC TTT TTT CAA CTT GAA ATA GCC TCC AAA T-3'). HEK293 cells were cotransfected with $1 \mathrm{mg}$ of p3'UTRTCF-4 and with p30UTRmut-TCF-4 plasmids and $1 \mathrm{mg}$ of a Renilla luciferase expression construct,pRL-TK (Promega Corporation), using Lipofectamine ${ }^{\mathrm{TM}} 2000$ reagent. Cells were harvested $24 \mathrm{~h}$ post-transfection and assayed with Dual-Luciferase assay (Promega Corporation) according to the manufacturer's instructions. Three independent experiments were performed in triplicate.

Western blotting. For whole protein extracts, liver tissue samples after grinding were homogenized in lysis buffer (Promega Corporation), incubated for $30 \mathrm{~min}$ on ice, and then centrifuged for $15 \mathrm{~min}$ at $14,000 \mathrm{x} \mathrm{g}$. Prior to use, all buffers were treated with a protease inhibitor cocktail (Konchem, China). Equal amounts of protein were separated discontinuously on 12-15\% SDS-PAGE and transferred to a PVDF membrane (Millipore, USA). The antibodies employed included anti-TCF-4, anti-cyclin D1, anti-c-Myc, anti-MMP-2,
anti-MMP-9 (all from Abcam, Cambridge, MA, USA) and GAPDH (Santa Cruz Biotechnology, Inc., Santa Cruz, CA, USA). Immunoblots were developed using anti-mouse or antirabbit HRP secondary antibodies (Santa Cruz Biotechnology, Inc.), followed by detection with Immobilon Western Blotting Chemiluminescent HRP Substrate (Millipore) according to the manufacturer's instructions by FX Fusion (Vilbert, France). For all western blottings, GAPDH was used as a reference gene.

Nude mouse tumor xenograft model and miR-139 treatment. HepG2 HCC cells were subcutaneously injected into 5-week-old female nude mice (Cancer Institute of the Chinese Academy of Medical Science). When the tumor volume reached $50 \mathrm{~mm}^{3}$, mice were randomly divided into 2 groups (8 mice/group). Each group was treated with miR-139 or a miR-CON oligo in $100 \mu \mathrm{l}$ Lipofectamine through local injection of the xenograft tumor at multiple sites. The treatment was performed once every 3 days for 24 days. The tumor volume was measured with a caliper twice/week, using the following formula: Tumor size $=a b^{2} / 2$, where $a$ is the larger and $b$ is the smaller of the 2 dimensions.

Statistical analysis. Student's t-test and one-way analysis of variance were used to determine statistical significance. All error bars represent the standard error of the mean. Pearson's correlation coefficient was calculated to test the association between miR-139 and TCF-4 in the normal vs. tumor groups. Statistical significance for all the tests, as assessed by calculating the $\mathrm{P}$-value, was $<0.05$.

\section{Results}

miR-139 is downregulated in HCC tissues and cancer cells. In the present study, we aimed to ascertain whether miR-139 has an effect on HCC. miR-139 expression in primary HCC tumors and normal liver tissues was assessed by qRT-PCR. Fig. 1A shows that miR-139 was highly expressed in normal liver 

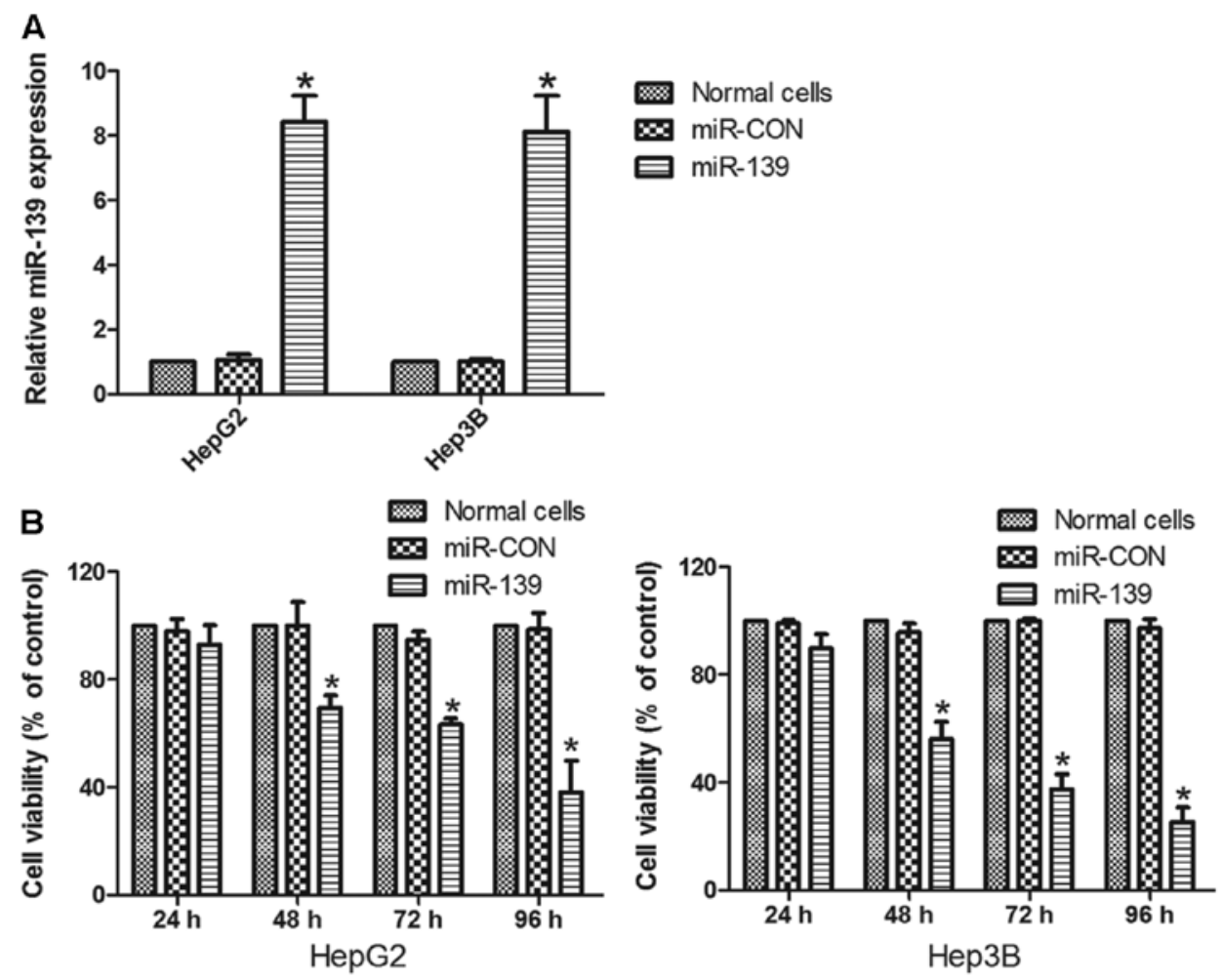

C

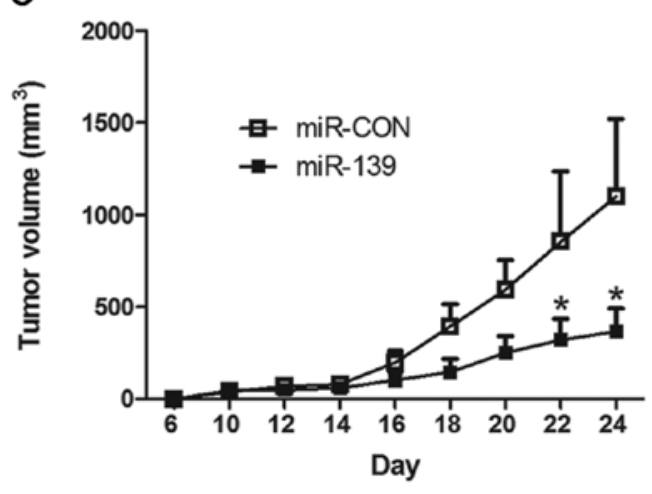

Figure 2. Overexpression of miR-139 suppresses HCC cell proliferation. (A) miR-139 expression was quantified by qRT-PCR analysis. Expression of miR-139 was significantly increased in both HepG2 and Hep3B cells transfected with miR-139 relative to the miR-CON and normal cells. ("P<0.05). (B) Cellular viability assay. MTT assay of HepG2 and Hep3B cells following mock transfection or transfection with miR-CON or miR-139 ("P<0.05). (C) Tumor volume growth curves of HepG2 tumors in mice treated with miR-139 $(n=8)$ or miR-CON $(n=8)$. miR-CON refers to the control oligonucleotides. " $\mathrm{P}<0.05$. HCC, hepatocellular carcinoma.

tissues when compared with the expression in the low-grade primary HCC tumors. We also examined the expression levels of miR-139 in the HCC cell lines HepG2, SMMC7721, Hep3B and in the normal liver cell line HL7702 (Fig. 1B). These cell lines demonstrated the same expression patterns as miR-139 in the liver sample tissues.

Upregulation of miR-139 inhibits HCC cell proliferation in vitro and in vivo. To explore the role of miR-139 in proliferation, we used an overexpression approach. An miR-139 oligonucleotide was used to overexpress miR-139 in HepG2 and Hep3B cells. Transient transfection of miR-139 led to the upregulation of miR-139 expression as determined by qRT-PCR (Fig. 2A). A significant decrease in cell viability was observed over time in HCC cells that exhibited high miR-139 expression (Fig. 2B) when compared to the cells expressing control levels of $\mathrm{miR}$ (miR-CON) as determined by the MTT assay. Furthermore, overexpression of miR-139 significantly suppressed the tumorigenicity of HCC cells in nude mice (Fig. 2C). These observations suggest that miR-139 upregulation suppresses the proliferation of HCC in vitro and in vivo.

miR-139 overexpression inhibits invasion and induces apoptosis in HCC cells. To assess the role of miR-139 in the cell invasion of HCC, we used Transwell assays. miR-139 overexpression decreased the invasiveness of HepG2 and Hep3B cells when compared to the miR-CON (Fig. 3A and B). In addition, we assessed apoptosis in the control and miR-139-transfected cells by flow cytometric analysis of Annexin V-PI stained HepG2 and Hep3B cells (Fig. 3C and D). In both cell lines, the average apoptotic cell fractions (early apoptotic + apop- 

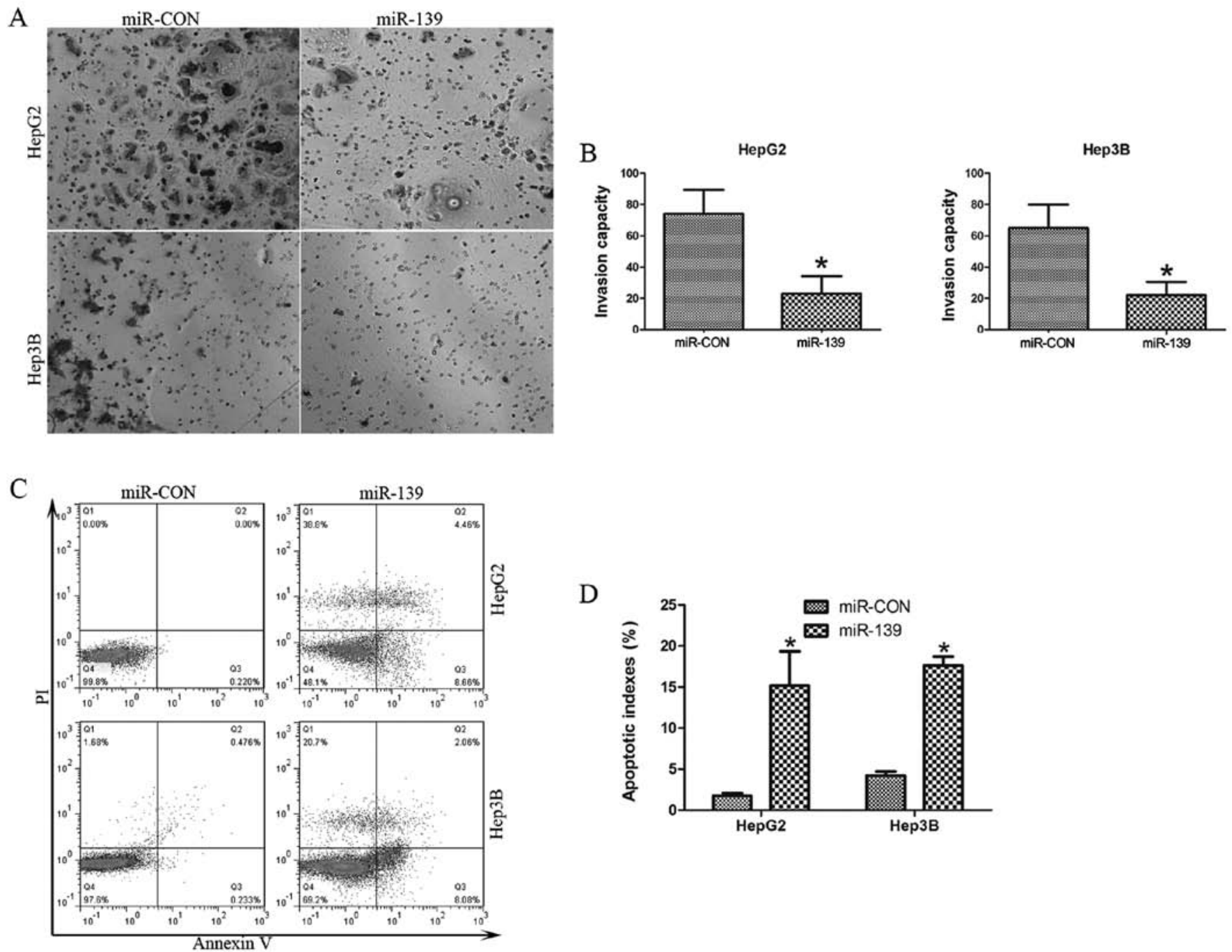

Figure 3. Overexpression of miR-139 suppresses the invasive capacity and induces apoptosis in HCC. (A and B) Invasion assay of HepG2 and Hep3B cells following miR-CON/miR-139 treatments. ${ }^{*} \mathrm{P}<0.05$. (C and D) Representation of an apoptosis assay in HepG2 and Hep3B cells after miR-CON/miR-139 treatments. ${ }^{*} \mathrm{P}<0.05$. HCC, hepatocellular carcinoma.

totic) were significantly increased following transfection with miR-139 when compared to the miR-CON $(\mathrm{P}<0.05)$. This finding points to a pro-apoptotic role of miR-139 and suggests that miR-139 affects apoptosis while regulating tumorigenicity. Together, these data demonstrate that miR-139 impacts the tumorigenic properties of HCC cells both in vitro and in vivo.

miR-139 downregulates TCF-4 expression by targeting its 3'UTR. To identify miR-139 targets, we performed a bioinformatics search (Targetscan, Pictar, RNhybrid) for putative mRNA targets of miR-139. Among the candidate targets, 3'UTR of human TCF-4 (nucleotides 5311-5324; NM_001243226) contained regions that matched the seed sequences of hsa-miR-139 (Fig. 4A). To verify that TCF-4 is a direct target of miR-139, TCF-4 3'UTR, containing the miR-139 binding sites, was cloned downstream of the luciferase open reading frame. These reporter constructs were used to transfect HEK293 cells, which express very low levels of miR-139 (Fig. 4B) and are highly transfectable. Increased expression of miR-139 upon transfection, as confirmed by qRT-PCR, significantly affected luciferase expression, measured as relative luciferase activity (Fig. 4C).

Conversely, when we performed the luciferase assays using a plasmid harboring the 3'UTR of TCF-4 mRNA, where the binding sites for miR-139 were inactivated by site-directed mutagenesis (miR-CON), we observed a consistent reduction in miR-139 inhibitory effect (Fig. 4C). To determine whether miR-139 affects TCF-4 expression in the HepG2 cellular environment, we analyzed the consequences of ectopic expression of miR-139 in HepG2 cells. Increased expression of miR-139 upon transfection was confirmed by qRT-PCR (Fig. 4D), and then the effects on endogenous levels of TCF-4 were analyzed by western blotting (Fig. 4E).

miR-139 overexpression significantly reduced the endogenous levels of TCF-4 when compared to HL7702 cells transfected with the scrambled miR-CON. Conversely, knockdown of miR-139 by anti-miR-139, confirmed by qRT-PCR (Fig. 4F) in HL7702 cells with high levels of endogenous miR-139, increased the protein levels of TCF-4 (Fig. 4G). 
A
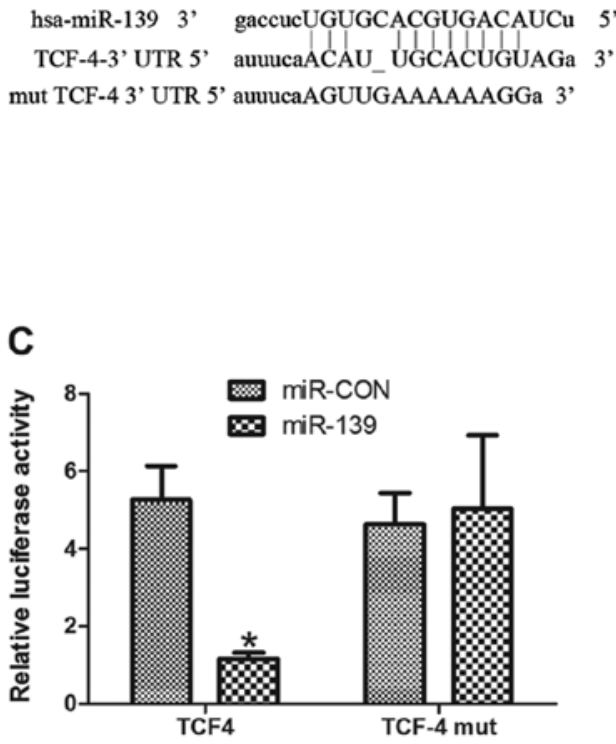

E

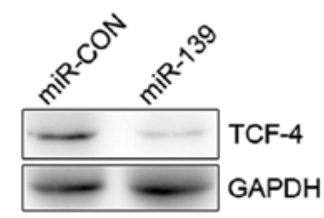

G

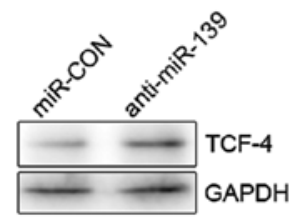

B

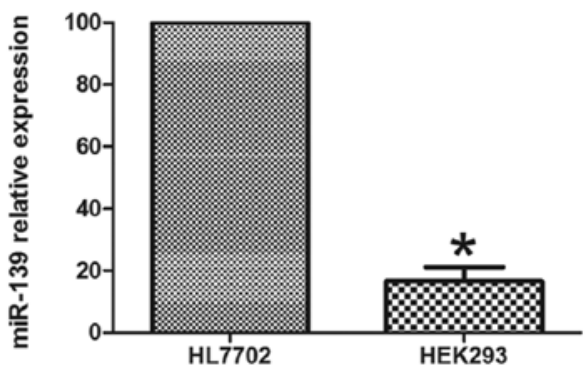

D

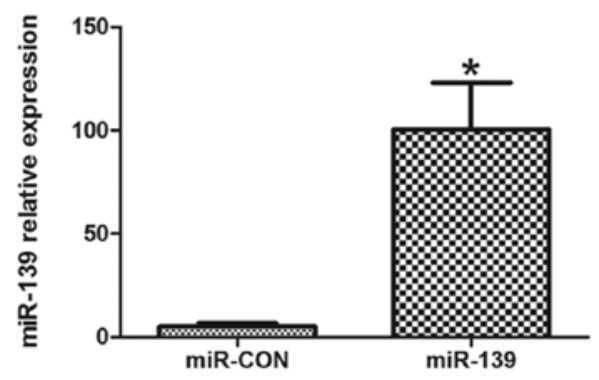

$\mathbf{F}$

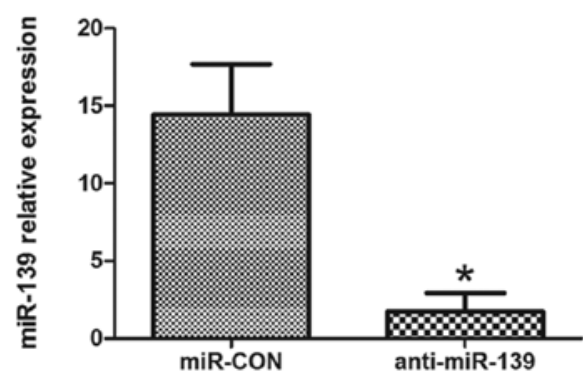

Figure 4. TCF-4 is a target gene of miR-139. (A) TCF-4 3'UTR contains 1 predicted miR-139 binding site. The alignment of the seed region of miR-139 with TCF-4 is indicated; the sites of target mutagenesis are indicated in red. (B) qRT-PCR of HEK293 cells after enforced expression of miR-139. (C) TCF-4 3'UTR is a target of miR-139. pGL3-TCF-4 luciferase construct, containing a wild-type or mutated TCF-4 3'UTR, was transfected into HEK293 cells. Relative suppression of firefly luciferase expression was standardized to a transfection control. The reporter assays were performed 3 times with essentially identical results. (D) qRT-PCR of HepG2 cells after enforced expression of miR-139. (E) Enforced expression of miR-139 decreases endogenous levels of TCF-4 protein in HepG2 cells. HepG2 cells were transfected with either miR-CON or miR-139 for $72 \mathrm{~h}$. TCF-4 expression was assessed by western blotting. Loading control was obtained using the anti-GAPDH antibody. (F) qRT-PCR showing downmodulation of miR-139 in HL7702 cells following anti-miR-139 transfection. (G) Western blotting showing TCF-4 expression after miR-139 downregulation using anti-miR-139. Anti-miR-139 was able to increase TCF-4 expression in the HL7702 cells. Data are presented as means \pm SD. *P<0.05. TCF-4, T-cell factor-4; 3'UTR, 3' untranslated region.

A

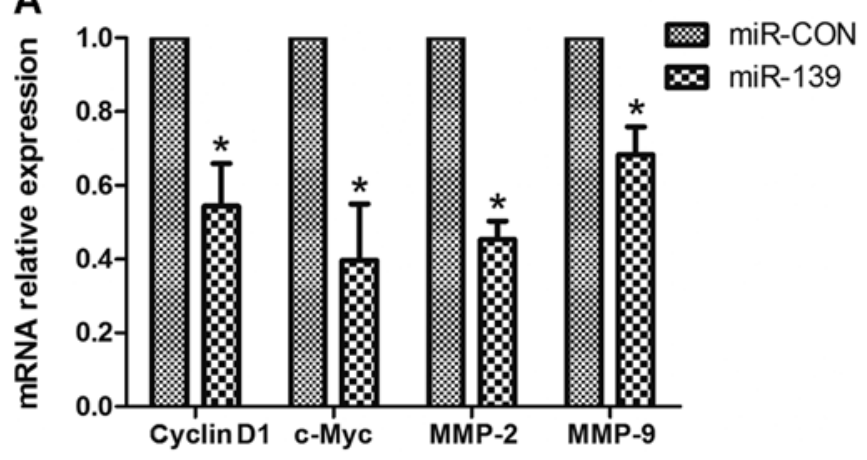

B

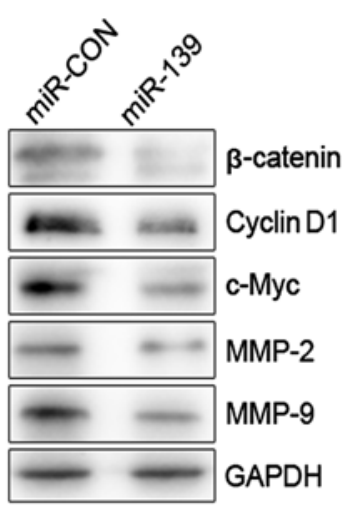

Figure 5. miR-139 overexpression suppresses $\beta$-catenin/TCF-4 signaling. (A) Expression of cyclin D1, c-Myc, MMP-2 and MMP-9 $48 \mathrm{~h}$ following transfection of HepG2 cells with miR-139 as detected by qRT-PCR. (B) Expression of $\beta$-catenin, cyclin D1, c-Myc, MMP-2 and MMP-9 48 h following transfection of HepG2 cells with miR-139 as detected by western blotting. TCF-4, T-cell factor-4. 
miR-139 overexpression suppresses the $\beta$-catenin/TCF-4 pathway. TCF- 4 binds to $\beta$-catenin to transactivate $\mathrm{Wnt}$ target genes (19). The essential role of the $\beta$-catenin/TCF-4 pathway as a mediator of miR-139 activity was confirmed by qRT-PCR and western blot assays which revealed reduced relative expression of $\beta$-catenin, cyclin D1, c-Myc, MMP-2 and MMP-9 in cells displaying high miR-139 expression (Fig. 5A and B). These data suggest critical downstream roles for the $\beta$-catenin/TCF-4 pathways in miR-139-mediated cell biology.

\section{Discussion}

miRNAs participate in maintaining the balance of gene regulating networks that determine the fate of cells. Bioinformatics analysis has revealed that miRNAs control the expression of one-third of the human proteome (23). Deregulation of miRNAs, which is a frequent outcome in human cancer, weakens this balance, thereby contributing to cancer progression. In the present study, we focused on miR-139, which has been found in head and neck (24), lung (25) and colorectal cancer (26), oral squamous cell carcinoma (27) and pancreatic cancer (28). However, no report on the biological consequences of miR-139 dysregulation in HCC have been characterized.

We focused on miR-139 as a potential candidate miRNA anti-oncogene in HCC. We confirmed miR-139 expression levels in clinical HCC tissues using qRT-PCR. The expression level of miR-139 was significantly low in HCC, as observed in our samples (Fig. 1). Based on these results, we hypothesized that miR-139 may play an important role in HCC. To test this hypothesis, we performed functional analyses to examine the function of miR-139. We analyzed cells that had been transfected with miR-139. As expected, high expression of miR-139 inhibited cell proliferation and invasion in HCC cells. Cell apoptosis was also dramatically induced following miR-139 transfected. These results suggest that miR-139 may function as an anticancer gene and plays an important role in the inhibition of cell growth and invasion of HCC.

We used the miRanda algorithm to identify targets of miR-139, and we identified TCF-4 a potential candidate target related to tumor-suppression. In this study, we focused on TCF-4, which was identified by study of WNT pathway signals. We performed a 3'UTR luciferase assay and observed that luciferase activity was increased after co-transfection with the miR-139 inhibitor and a 3'UTR vector containing the TCF-4 miR-139 target sequence. TCF-4 protein expression was also significantly upregulated in HepG2 and Hep3B cells that were transfected with the miR-139 inhibitor, indicating that TCF-4 is a direct target of miR-139.

The Wnt pathway is an important target for the development of novel therapeutics for cancer (29). Activated $\beta$-catenin/TCF- 4 signaling by accumulation of $\beta$-catenin in the nucleus has been implicated in human carcinogenesis, including HCC. This accumulation may result from mutation of the tumor-suppressor gene $\beta$-catenin itself. In the present study, we conducted a detailed mechanistic study to evaluate the efficacy of the inhibition of $\beta$-catenin/TCF- 4 signaling by miR-139 in HCC using HCC cell lines. We constructed miR-139 vectors in order to knockdown the expression of TCF-4. Western blot analysis revealed a decrease in TCF-4 protein expression in HepG 2 and Hep3B cells at 48 h postinfection with the respective miR-139 vectors. In addition, the miR-139 vector markedly suppressed $\beta$-catenin/TCF-4 transcriptional activity as well as the expression of 2 known target genes, Cyclin D1 and c-Myc, in HepG2 and Hep3B cells. These data indicate that miR-139 overexpression suppresses the $\beta$-catenin/TCF-4 activity in vitro.

In conclusion, this study revealed that the downregulation of miR-139 expression is a common event in HCC, and we provide evidence that miR-139 plays a role in regulating proliferation, invasion and apoptosis. miR-139 is able to inhibit TCF-4 protein expression through directly binding to the 3'UTR region of TCF-4 mRNA. The regulation of HCC growth, by upregulation of miR-139 expression may represent a novel therapeutic strategy for HCC.

\section{Acknowledgements}

This study was supported by the National Natural Science Foundation (No. 81071877), and the Fundamental Research Fund of Xi'an Jiaotong University (2010-01).

\section{References}

1. Cha C, DeMatteo RP and Blumgart LH: Surgery and ablative therapy for hepatocellular carcinoma. J Clin Gastroenterol 35 (Suppl 2): S130-S137, 2002.

2. Forner A, Llovet JM and Bruix J: Hepatocellular carcinoma. Lancet 379: 1245-1255, 2012.

3. Carr BI: Hepatocellular carcinoma: current management and future trends. Gastroenterology 127 (Suppl 1): S218-S224, 2004.

4. Cai X, Hagedorn $\mathrm{CH}$ and Cullen BR: Human microRNAs are processed from capped, polyadenylated transcripts that can also function as mRNAs. RNA 10: 1957-1966, 2004.

5. Lee Y, Kim M, Han J, et al: MicroRNA genes are transcribed by RNA polymerase II. EMBO J 23: 4051-4060, 2004.

6. Gregory RI, Yan KP, Amuthan G, et al: The microprocessor complex mediates the genesis of microRNAs. Nature 432: 235-240, 2004

7. Chendrimada TP, Gregory RI, Kumaraswamy E, et al: TRBP recruits the Dicer complex to Ago2 for microRNA processing and gene silencing. Nature 436: 740-744, 2005.

8. Han J, Lee Y, Yeom KH, et al: Molecular basis for the recognition of primary microRNAs by the Drosha-DGCR8 complex. Cell 125: 887-901, 2006.

9. Valencia-Sanchez MA, Liu J, Hannon GJ and Parker R: Control of translation and mRNA degradation by miRNAs and siRNAs. Genes Dev 20: 515-524, 2006.

10. Plasterk RH: Micro RNAs in animal development. Cell 124: 877-881, 2006.

11. Nusse R and Varmus HE: Many tumors induced by the mouse mammary tumor virus contain a provirus integrated in the same region of the host genome. Cell 31: 99-109, 1982.

12. Peifer $M$ and Polakis P: Wnt signaling in oncogenesis and embryogenesis - a look outside the nucleus. Science 287: 1606-1609, 2000.

13. Giles RH, van Es JH and Clevers H: Caught up in a Wnt storm: Wnt signaling in cancer. Biochim Biophys Acta 1653: 1-24, 2003.

14. Nelson WJ and Nusse R: Convergence of Wnt, $\beta$-catenin, and cadherin pathways. Science 303: 1483-1487, 2004.

15. Brantjes H, Barker N, van Es J and Clevers H: TCF: Lady Justice casting the final verdict on the outcome of Wnt signalling. Biol Chem 383: 255-261, 2002.

16. Hecht A, Vleminckx K, Stemmler MP, van Roy F and Kemler R: The $\mathrm{p} 300 / \mathrm{CBP}$ acetyltransferases function as transcriptional coactivators of $\beta$-catenin in vertebrates. EMBO J 19: 1839-1850, 2000.

17. Takemaru KI and Moon RT: The transcriptional coactivator CBP interacts with $\beta$-catenin to activate gene expression. J Cell Biol 149: 249-254, 2000. 
18. Kramps T, Peter O, Brunner E, et al: Wnt/wingless signaling requires BCL9/legless-mediated recruitment of Pygopus to the nuclear $\beta$-catenin-TCF complex. Cell 109: 47-60, 2002.

19. Graham TA, Ferkey DM, Mao F, Kimelman D and Xu W: Tcf4 can specifically recognize $\beta$-catenin using alternative conformations. Nat Struct Biol 8: 1048-1052, 2001.

20. Hoverter NP and Waterman ML: A Wnt-fall for gene regulation: repression. Sci Signal 1: pe43, 2008.

21. El-Tanani MK, Barraclough R, Wilkinson MC and Rudland PS: Regulatory region of metastasis-inducing DNA is the binding site for T cell factor-4. Oncogene 20: 1793-1797, 2001.

22. El-Tanani M, Barraclough R, Wilkinson MC and Rudland PS: Metastasis-inducing DNA regulates the expression of the osteopontin gene by binding the transcription factor Tcf- 4 . Cancer Res 61: 5619-5629, 2001.

23. Lewis BP, Burge CB and Bartel DP: Conserved seed pairing, often flanked by adenosines, indicates that thousands of human genes are microRNA targets. Cell 120: 15-20, 2005.

24. Wong TS, Liu XB, Wong BY, Ng RW, Yuen AP and Wei WI: Mature miR-184 as potential oncogenic microRNA of squamous cell carcinoma of tongue. Clin Cancer Res 14: 2588-2592, 2008.
25. Mascaux C, Laes JF, Anthoine G, et al: Evolution of microRNA expression during human bronchial squamous carcinogenesis. Eur Respir J 33: 352-359, 2009.

26. Arndt GM, Dossey L, Cullen LM, et al: Characterization of global microRNA expression reveals oncogenic potential of miR-145 in metastatic colorectal cancer. BMC Cancer 9: 374, 2009.

27. Kozaki K, Imoto I, Mogi S, Omura K and Inazawa J: Exploration of tumor-suppressive microRNAs silenced by DNA hypermethylation in oral cancer. Cancer Res 68: 2094-2105, 2008.

28. Lee EJ, Gusev Y, Jiang J, et al: Expression profiling identifies microRNA signature in pancreatic cancer. Int J Cancer 120: 1046-1054, 2007.

29. Takahashi-Yanaga $\mathrm{F}$ and Kahn M: Targeting Wnt signaling: can we safely eradicate cancer stem cells? Clin Cancer Res 16: 3153-3162, 2010. 